\title{
Iterative Decoding and Soft Interference Cancellation in Fast Frequency Hopping Multiuser System Using Clipped Combining
}

\author{
Sohail Ahmed, Soon Xin Ng, Lie-Liang Yang and Lajos Hanzo \\ School of ECS, University of Southampton, SO17 1BJ, UK. \\ Tel: +44-23-8059 3364, Fax: +44-23-8059 4508 \\ Email: \{sa03r,sxn,lly,lh\}@ecs.soton.ac.uk; http://www-mobile.ecs.soton.ac.uk
}

\begin{abstract}
Iterative decoding (ID) aided fast frequency hopping (FFH), $M$-ary frequency shift keying (MFSK) using clipped combining in multiple access (MA) channels is investigated. All users' data are convolutionally encoded and the encoded bits are interleaved and converted to $M$-ary symbols, which are transmitted using FFH-MFSK modulation. The soft metrics to be passed from the demodulator to the decoder are derived assuming a Rayleigh fading channel. We also propose a novel multiuser detection (MUD) scheme which employs joint soft decoding as well as successive interference cancellation (SIC), the receiver exploiting the soft information fed back by the decoder to the demodulator in order to cancel the interference imposed by reliable symbols. Our simulation results show that the proposed scheme is capable of combatting multiuser interference and outperforms the conventional ID by about $3 \mathrm{~dB}$.
\end{abstract}

\section{INTRODUCTION}

In fast frequency hopping (FFH), $M$-ary frequency shift keying (MFSK) systems operating in a multiple access (MA) scenario [1], narrowband interference imposed by other users is the main source of performance degradation [2]. In a conventional cellular system [3], the base station's (BS) receiver usually employs a separate single-user detection (SUD) receiver for each user [2]. However, given the knowledge of all users' addresses, the BS's receiver may invoke Multiuser Detection (MUD) [4], which jointly detects all users' signals. Specifically, when a multi-stage Successive Interference Cancellation (SIC) assisted MUD [2] is employed, the effects of symbols reliably detected at a specific SIC stage can be removed in the subsequent stages, thereby reducing the interference constituted by these reliably detected users.

A plethora of work has been contributed towards joint MUD and Soft-Input-Soft-Output (SISO) decoding designed for Direct Sequence Code Division Multiple Access (DSCDMA) [5], [6]. The basic idea behind soft-cancellation assisted iterative MUD, also referred to as turbo MUD [6] is to carry out demodulation/decoding in multiple stages. More specifically, in coherently detected DS-CDMA, estimates of the dispersive channel are generated, which facilitate the cancellation of the exact interference imposed by reliably decoded symbol on the composite multiuser signal. This remodulated

The financial support of the EPSRC, UK and EU under the auspices of the Phoenix and Newcom projects and of Higher Education Commission, Pakistan is gratefully acknowledged. signal estimate can then be subtracted from the received signal. By contrast, frequency hopping $(\mathrm{FH})$ systems are typically employed in conjunction with noncoherently detected MFSK. Thus, in the context of FH-MFSK the lack of knowledge concerning the channel conditions prohibits the employment of classic SIC based MUD schemes typically employed in DSCDMA [4], [5], [6]. Consequently, the idea of joint decoding and MUD has attracted limited attention as far as FH-MFSK systems are concerned.

Nonetheless, some soft metrics and detection algorithms have been proposed in [7], [8], [9] for employment in slowand fast-FH. In the context of combatting multiuser interference (MUI), Fiebig and Robertson [10] employed soft decision decoded convolutional, turbo and RS codes, although no attempt was made to exploit the decoder's soft output. Park and Lee [11] extended the work reported in [10] employing Iterative Decoding (ID). The concept of iterative soft information exchange between an $M$-ary orthogonal demodulator and a SISO decoder has been investigated in [12], [13].

In this contribution, we employ for the first time the concept of soft information assisted MUD in FFH-MFSK and introduce a novel MUD scheme that invokes joint SISO decoding combined with SIC for mitigating the effects of MUI. We demonstrate the power of the proposed algorithm using binary convolutional codes. In the receiver, we employ clipped or Soft-Limiter Combining (SLC) [1] and SIC based on feedback from the SISO decoder. The soft metrics passed by the MFSK demodulator to the decoder are also derived, assuming uncorrelated Rayleigh fading.

The rest of this paper is structured as follows. In Section II, the system under consideration is described and in Section III, the soft metrics are derived and the proposed Soft-SIC scheme is discussed. In Section IV, we will demonstrate with the aid of simulation results that the proposed SIC method results in useful performance gains. Finally, in Section V, we present our conclusions.

\section{SySTEM DESCRIPTION}

The FFH-MFSK system considered consists of a single cell, serving multiple users with the aid of a BS and utilizing a spread spectrum bandwidth of $W_{s s}$. The schematic of the proposed system is depicted in Fig. 1. All users' binary 


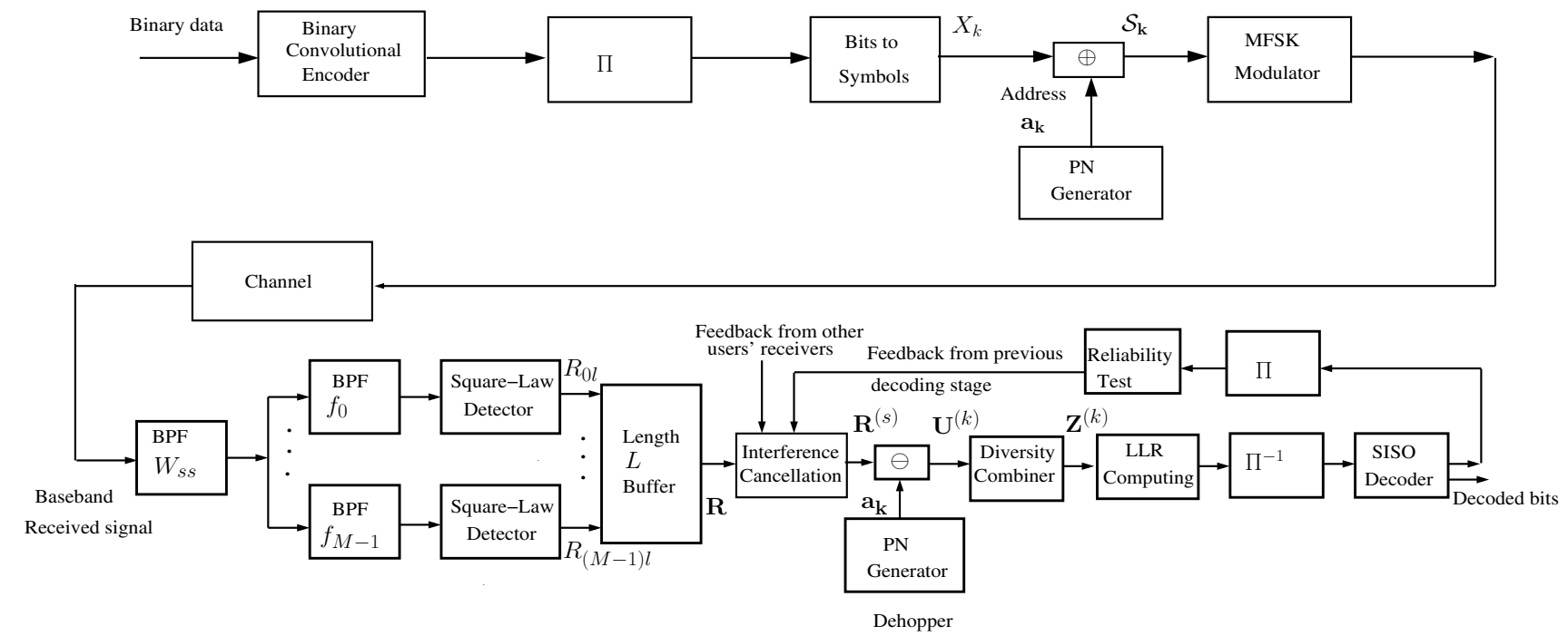

Fig. 1. Block diagram of the FFH-MFSK system for the $k$ th user, $k=1,2, \ldots, N_{U}$, employing SISO convolutional decoding and SIC.

data are encoded by a binary non-recursive, Non-Systematic Convolutional (NSC) [14] code before bit interleaving. Following bit interleaving, the NSC coded bits are converted to $M$-ary symbols as shown in Fig. 1 . The $k$ th user, $k=$ $1,2, \ldots, N_{U}$, is assigned a unique and random $L$-tuple $\mathrm{FFH}$ address $\mathbf{a}_{\mathbf{k}}=\left[a_{k}(0), a_{k}(1), \ldots, a_{k}(L-1)\right]$, which is output by the PN generator seen in Fig. 1, where $a_{k}(l) \in \mathrm{GF}(M)$, $l=0,1, \ldots, L-1, L$ is the number of frequency hops per symbol and $\mathrm{GF}(M)$ represents a Galois field having $M$ elements. In the MFSK modulator of Fig. 1, an MFSK tone is chosen for transmission whose frequency during the $l$ th hop of duration $T_{h}$ is chosen according to [2]

$$
\mathcal{S}_{\mathbf{k}}=\left[\mathcal{S}_{k}(0), \mathcal{S}_{k}(1), \ldots, \mathcal{S}_{k}(L-1)\right]=X_{k} \cdot \mathbf{1} \oplus \mathbf{a}_{\mathbf{k}},
$$

where 1 denotes a unit vector of length $L, X_{k}$ is the $M$ ary symbol to be transmitted by the $k$ th user and $\oplus$ denotes addition in the Galois Field. Note that $\mathcal{S}_{k}(l), a_{k}, X_{k} \in$ $[0,1, \ldots, M-1]$. We assume that the bandwidth occupied by the signal transmitted during each FFH chip interval is given by $R_{h}=1 / T_{h}$. The frequency separation between adjacent FH tones is also assumed to be $R_{h}$. Thus, the orthogonality of the FFH frequency tones is maintained. Note that for ease of analysis, random FFH patterns are assumed [2].

The channel is modeled by uncorrelated, frequency-flat Rayleigh fading for each transmitted frequency. We assume that the frequency separation between the adjacent signalling frequencies is higher than the coherence bandwidth of the channel. Thus each signalling tone can be assumed to experience independent fading. The fading envelopes experienced by the various users are also independent of each other. Furthermore, the transmitted signals are corrupted by Additive White Gaussian Noise (AWGN) having a one-sided power spectral density of $N_{0}$.

When a particular user transmits a signal to the BS, the signals transmitted by all other users may be modeled as continuous-wave interfering tones. For the sake of simplicity, we also assume a chip-synchronous system, where the chip transitions of all users are aligned in time. Furthermore, perfect power control is assumed, hence the power of all the signals received by the BS from all users is identical.

The BS receiver's schematic is also shown in Fig. 1, where we assume that the hopping sequence of the FFH demodulator is in synchronism with that of the FFH modulator of the transmitter. After carrier demodulation, a bank of $M$ squarelaw detectors detect the energy received in each MFSK tone. The outputs of the detectors are dehopped and combined over $L$ hops using SLC, as will be discussed in the next section. Then soft decoding and SIC is employed for cancelling the multiuser interference (MUI), which is also discussed in the context of the proposed SIC scheme in the next section.

\section{Soft Information BASED SIC SCHEME}

As seen in Fig. 1, the decoder accepts soft inputs from the demodulator, employs the Maximum A Posteriori (MAP) algorithm [14] and delivers soft a posteriori information as well as the decoded bits. In the following section we discuss how soft information is derived by the demodulator from the channel observations.

\section{A. Soft Decoder Input and Iterative Decoding}

The iterative soft information exchange between an $M$ ary orthogonal demodulator and a SISO binary decoder has been considered in [12], [13]. We apply this concept in the context of the proposed FFH-MFSK receiver and refer to it as the SUD-ID scheme, where the term SUD implies that no attempt has been made to detect all users' symbols jointly. In a conventional SISO decoding system, the demodulator inputs the Log-Likelihood Ratios (LLRs) [14] of the received signal to the decoder. In order to compute the LLRs for the $k$ th user, we need the probability that the $m t h$ symbol $X_{k}=m$ was transmitted, $m=0, \ldots, M-1$, given that the signal $\mathbf{Z}^{(k)}$, which represents the set of $M$ outputs of the diversity 
combiners of Fig. 1, is received. This probability is given by

$$
P^{(k)}\left(X_{k}=m \mid \mathbf{Z}^{(k)}\right)=\frac{p^{(k)}\left(\mathbf{Z}^{(k)} \mid X_{k}=m\right) P^{(k)}\left(X_{k}=m\right)}{p^{(k)}\left(\mathbf{Z}^{(k)}\right)},
$$

where $p^{(k)}\left(\mathbf{Z}^{(k)} \mid X_{k}=m\right)$ is the PDF of the received signal $\mathbf{Z}^{(k)}$, given that $X_{k}=m$ is transmitted. Furthermore, $P^{(k)}\left(X_{k}=m\right)$ is the a priori probability of the symbol $X_{k}=m$, while $p^{(k)}\left(\mathbf{Z}^{(k)}\right)$ is the probability of receiving signal $\mathbf{Z}^{(k)}$, which is given by

$$
p^{(k)}\left(\mathbf{Z}^{(k)}\right)=\sum_{m=0}^{M-1} p^{(k)}\left(\mathbf{Z}^{(k)} \mid X_{k}=m\right) P^{(k)}\left(X_{k}=m\right) .
$$

The probability given by (3) is a constant for a given received signal $\mathbf{Z}^{(k)}$. Moreover, for equiprobable symbols, we have $P^{(k)}\left(X_{k}=m\right)=1 / M$. Hence, the PDF $p^{(k)}\left(\mathbf{Z}^{(k)} \mid X_{k}=m\right)$ uniquely and unambiguously describes the statistics required for estimating the probability $P^{(k)}\left(X_{k}=m \mid \mathbf{Z}^{(k)}\right)$. For independent fading of all tones, the $\operatorname{PDF} p^{(k)}\left(\mathbf{Z}^{(k)} \mid X_{k}=m\right)$ is given by

$$
\begin{aligned}
p^{(k)}\left(\mathbf{Z}^{(k)} \mid X_{k}=m\right) & =f_{z_{m}}^{(k)}\left(x_{m} \mid X_{k}=m\right) \\
& \times \prod_{n=0, n \neq m}^{M-1} f_{z_{n}}^{(k)}\left(x_{n} \mid X_{k}=m\right),
\end{aligned}
$$

where $f_{z_{n}}^{(k)}\left(x_{n} \mid X_{k}=m\right)$ represents the PDF of the output of the $n$th diversity combiner, $n=0,1, \ldots, M-1$, given that $X_{k}=m$ is transmitted.

In order to derive the PDFs of the diversity combiner outputs, we first consider the PDFs of the square-law detector outputs before diversity combining. Although the signal transmitted by a specific user may be interferred by other active users' signals, it is challenging to derive the PDF of the square-law detector output considering all possible combinations of the interfering tones. Hence, we derive the soft information from the channel observations assuming a somewhat simplistic but tractable interference-free channel. Moreover, although clipped combining is employed in the receiver, as discussed in the following section, we perform the forthcoming analysis assuming linear combining, using no clipping at the receiver. This assumption has been stipulated for further simplifying the analysis and is supported by the observation that clipping is an operation performed to reduce effects of MUI. Hence, our analysis may result in sub-optimal soft information, but we will nevertheless demonstrate in Section IV, that valuable performance improvements can be achieved using this approach.

Assuming that $X_{k}=m$ is transmitted by the $k$ th user in the $l$ th hop, it can be readily shown that for independent Rayleigh fading, the PDF of the noise-normalized square-law detector output $U_{m l}^{(k)}$ after dehopping as seen in Fig. 1, is given by [3]

$$
f_{U_{m l}^{(k)}}\left(y_{m} \mid X_{k}=m\right)=\frac{1}{1+\gamma_{h}} e^{\frac{-y_{m}}{1+\gamma_{h}}}, \quad y_{m} \geq 0,
$$

where $\gamma_{h}=b R_{c} E_{b} /\left(N_{0} L\right)$ is the Signal-to-Noise Ratio (SNR) per hop, $R_{c}$ is the code rate, $E_{b}$ is the transmitted energy per bit and $b$ is the number of bits per symbol.
Similarly, for all the interfering or non-signal tones, $n=$ $0,1, \ldots, M-1, n \neq m$, the corresponding PDF is given by

$$
f_{U_{n l}^{(k)}}\left(y_{n} \mid X_{k}=m\right)=e^{-y_{n}}, \quad y_{n} \geq 0 .
$$

From (5), using the Characteristic Function (CF) approach [3] ${ }^{1}$, we can derive the PDF of the linear combiner output $Z_{m}^{(k)}$ seen in Fig. 1, which can be expressed as

$$
f_{Z_{m}^{(k)}}\left(x_{m} \mid X_{k}=m\right)=\frac{x_{m}^{L-1}}{\left(1+\gamma_{h}\right)^{L} \Gamma(L)} e^{-x_{m} /\left(1+\gamma_{h}\right)} .
$$

Similarly, for all the non-signal tones, $n=0,1, \ldots, M-1, n \neq$ $m$ we have

$$
f_{Z_{n}^{(k)}}\left(x_{n} \mid X_{k}=m\right)=\frac{x_{n}^{L-1}}{\Gamma(L)} e^{-x_{n}} .
$$

Inserting (7) and (8) in (4) and after further simplifications, we have

$$
\begin{aligned}
p^{(k)}\left(\mathbf{Z}^{(k)} \mid X_{k}=m\right) & =\left[\frac{1}{\left(1+\gamma_{h}\right)^{L}} \frac{1}{\Gamma^{M}(L)} \prod_{n=0}^{M-1} x_{n}^{L-1} e^{-x_{n}}\right] \\
& \times \exp \left(\frac{x_{m} \gamma_{h}}{1+\gamma_{h}}\right) .
\end{aligned}
$$

We can see in (9) that all the terms, except the last exponential term are common, for any of the $m$ th symbol, $m=0,1, \ldots, M-1$. Since the computation of the LLRs requires the logarithm of the bit probabilities, we consider the common terms as a normalization factor and express the normalized probability $p\left(\mathbf{Z}^{(k)} \mid X_{k}=m\right)$ as

$$
p^{(k)}\left(\mathbf{Z}^{(k)} \mid X_{k}=m\right)=\exp \left(\frac{x_{m} \gamma_{h}}{1+\gamma_{h}}\right) .
$$

Upon inserting (10) in (2), we can derive the corresponding normalized symbol probabilities. Finally, the bit probabilities can be derived from the symbol probabilities [10], [12] and using the bit probablities, the LLRs can be computed [14]. The decoder now outputs a posteriori information, which may be fed back to the demodulator as a priori information, for further assisting the demodulator to improve its soft information output. In conventional ID, this exchange of soft information between the demodulator and decoder may be invoked a number of times [14].

\section{B. Soft-SIC Algorithm}

Let us now outline our proposed Soft-SIC algorithm. Based on the LLRs derived above, the joint Soft-SIC and SISO decoding process may be described as follows:

1) As seen in Fig. 1, the matrix $\mathbf{R}$ hosting the square-law detector outputs is separately dehopped by each receiver using the corresponding unique user address, resulting in the matrix $\mathbf{U}^{(k)}, k=1,2, \ldots, N_{U}$, for the $k$ th user. The dehopping process may be expressed as

$$
\begin{aligned}
& U_{p l}^{(k)}=R_{m l}, \text { given } p=m \ominus a_{k}(l), \\
& m, p=0,1, \ldots, M-1 ; l=0,1, \ldots, L-1 .
\end{aligned}
$$

\footnotetext{
${ }^{1}$ The $\mathrm{CF}$ is the Fourier transform of the PDF and the fact that $\mathrm{CF}$ of a sum of random variables is the product of their individual CFs [3] has been exploited here.
} 
2) SLC is performed on the dehopped signals of Fig. 1, resulting in $M$ decision variables for each of the users. The clipping operation may be expressed by [1]

$$
f\left(U_{m l}^{(k)}\right)= \begin{cases}C, & \text { if } U_{m l}^{(k)} \geq C \\ U_{m l}^{(k)}, & \text { otherwise }\end{cases}
$$

where $C$ represents an appropriately chosen clipping threshold. The decision variable recorded after clipped combining for the $k$ th user is given by [1]

$$
Z_{m}^{(k)}=\sum_{l=0}^{L-1} f\left(U_{m l}^{(k)}\right), \quad m=0,1, \ldots, M-1 .
$$

3) From the diversity combiner outputs of Fig. 1, the soft information, as derived above, is passed as a priori input to the SISO decoder, which generates the a posteriori LLRs. The a posteriori LLRs are then converted to equivalent bit probabilities and then to symbol probabilities [10] $P_{a p o}^{(k)}\left(X_{k}=m \mid \mathbf{Z}^{(k)}\right), m=0,1, \ldots, M-1$. 4) Next, as seen in Fig. 1 a reliability test of all users' symbols is carried out. Specifically, if the ratio of the second largest to the largest of the $M$ number of a posteriori probabilites $P_{\text {apo }}^{(k)}\left(X_{k}=m \mid \mathbf{Z}^{(k)}\right)$ corresponding to a symbol is less than a predefined threshold $\epsilon_{T}$, that is, if we have

$$
\epsilon^{(k)}=\frac{\max _{2}\left[P_{a p o}^{(k)}\left(X_{k}=m \mid \mathbf{Z}^{(k)}\right)\right]}{\max \left[P_{a p o}^{(k)}\left(X_{k}=m \mid \mathbf{Z}^{(k)}\right)\right]}<\epsilon_{T},
$$

then the symbol is declared reliable, else it is deemed unreliable. The reliable symbols are estimated from the $a$ posteriori symbol probabilities using conventional hard decisions. Specifically, if the $k$ th user's symbol, $k=$ $1,2, \ldots, N_{U}$ satisfies the reliability test given in (14), then $X_{k}^{(d)}$ is the estimate of the $k$ th user's symbol, where $X_{k}^{(d)}=0,1, \ldots, M-1$, is the index of the maximum of $P_{a p o}^{(k)}\left(X_{k}=m \mid \mathbf{Z}^{(k)}\right)$. If no user passes the test, we earmark that specific user's symbol for subsequent erasure, which has the lowest value of $\epsilon$.

5) If some but not all symbols are reliably detected, we proceed with the $s$ th stage of SIC, where $s=1,2, \ldots$. We also define $\mathbf{R}^{(s)}$ as the modified form of $\mathbf{R}$ at the $s$ th stage, as seen in Fig. 1. The sth stage of the SIC, $s \geq 1$, consists of the following steps:

a) Modify the matrix $\mathbf{R}^{(s-1)}$ by erasing the elements representing the signals, which correspond to all the symbols that were declared reliable in the $(s-$ 1)th stage (Step 4). Hence, if $X_{k}^{(d)}$ is the estimated symbol of the $k$ th user, then, after interference cancellation, we have

$$
R_{m l}^{(s)}= \begin{cases}0 & \text { for } m=X_{k}^{(d)} \oplus a_{k}(l) \\ R_{m l}^{(s-1)} & \text { otherwise. }\end{cases}
$$

b) Repeat Steps 1 to 4 outlined above for the matrix $\mathbf{R}^{(s)}$, to determine if more symbols can be declared reliable. In each SIC iteration, for symbols already declared reliable, use the symbol probabilities $P^{(k)}\left(X_{k}=m \mid \mathbf{Z}^{(k)}\right), m=0,1, \ldots, M-1$ obtained in the specific SIC stage in which they were declared reliable. For the remaining unreliable symbols, new values of symbol probabilities, bit probabilities and LLRs will be obtained after each iteration. The SIC iterations can be continued until all users' symbols meet the reliability test or, until the affordable number $S$ of SIC stages have been completed.

6) After all symbols have satisfied the reliability test, or alternatively, when S number of SIC iterations have been completed, we perform convolutional hard decoding using the symbol probabilities and LLRs obtained at the end of last SIC stage.

It can be observed from our discussions of the Soft-SIC scheme that the cancellation of the symbols declared reliable in a specific stage may lead to inadvertent cancellation of hitherto undetected symbols, if two users happen to transmit the same FFH tone. This might lead to erroneous decisions and to subsequent propagation of decision errors. However, the probability of this event is typically low and we will show in the next section that the proposed SIC scheme yields a useful overall BER performance improvement. Another drawback of the proposed scheme is that the optimum test threshold $\epsilon$ defined in (14) has to be determined, which may depend upon the SNR, the number of users, as well as the modulation and diversity order.

\section{Simulation Results and Discussion}

In this section we present our BER performance results for the SUD-ID scheme and the Soft-SIC scheme discussed in Section III, when they are employed in a FFH-MFSK system operating in a frequency-flat Rayleigh fading MA channel. Unless otherwise stated, a maximum of three SIC stages per MFSK symbol have been allowed, after which the detection process is concluded, i.e. we have $S=3$. For the sake of fair comparisons, we also invoke three decoding iterations in the context of the SUD-ID scheme. Additionally, optimum clipping thresholds $C$ and the test thresholds $\epsilon$, defined in Section III, have been employed in all simulations. Unless otherwise stated, we employ a $1 / 2$ rate NSC code with constraint length of 3 characterised by octal generator polynomial of $(7,5)$, and the coded bits are interleaved by a $(70 \times 40)$-bit block interleaver.

Fig. 2 shows the BER versus SNR performance of the SoftSIC and SUD-ID schemes, assuming $L=6, M=32$ and $N_{U}=15$. The performance of the SUD using SLC and Soft Decision Decoding (SDD) but no ID, which is referred to as the SUD-SDD scheme, has also been shown in Fig. 2. Similarly, the performance of the same system using hard decision decoding (HDD), referred to as the SUD-HDD scheme, is also shown. Moreover, as benchmark, the single user (interferencefree) performance of the FFH-MFSK scheme using ID is also included. The results of Fig. 2 demonstrate that the SDD yields significantly better performance than the HDD, thus validating the benefits of our soft information derived in Section III-A. Moreover, at around $E_{b} / N_{0}=18 d B$, the Soft-SIC scheme attains a near-zero BER, which is about $8 \mathrm{~dB}$ lower than the 


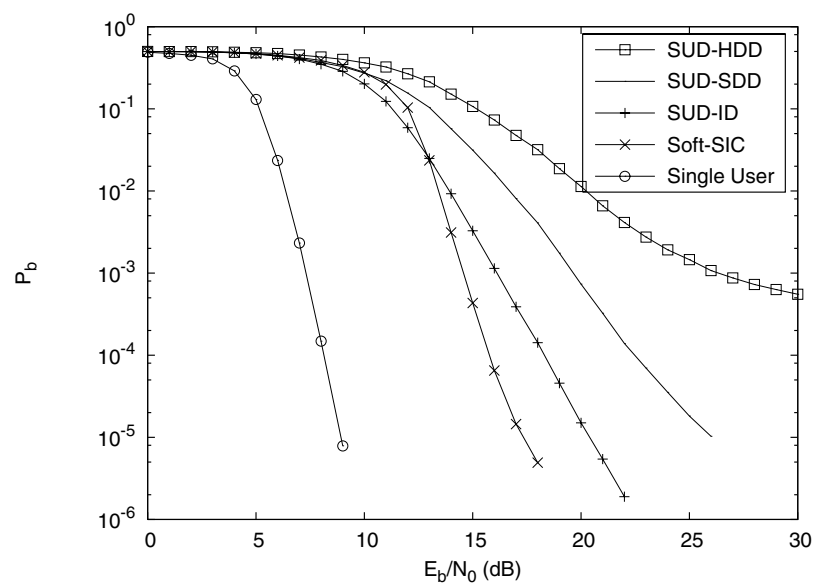

Fig. 2. BER versus SNR performance for SIC and ID schemes employed in soft decoded FFH-MFSK SLC receiver communicating over a Rayleigh fading channel, assuming $L=6, M=32$, and $N_{U}=15$.

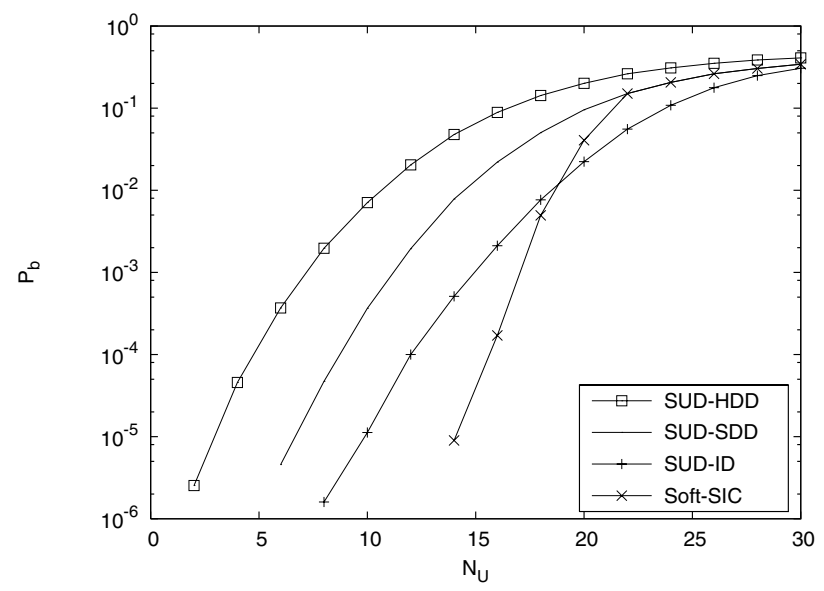

Fig. 3. BER versus number of users performance for SIC and ID schemes employed in soft decoded FFH-MFSK SLC receiver communicating over a Rayleigh fading channel, assuming $L=7, M=32$ and $E_{b} / N_{0}=15 \mathrm{~dB}$.

$E_{b} / N_{0}$ value required by the SUD-SDD scheme, although it is $8 \mathrm{~dB}$ higher than that of the single user scenario. We also note in Fig. 2 that our proposed scheme ourperforms the conventional SUD-ID scheme beyond the point of $E_{b} / N_{0}=$ $14 d B$. For $E_{b} / N_{0}<14 d B$, the Soft-SIC scheme is inferior in comparison to the SUD-ID scheme, indicating that at low SNR values the SIC scheme is ineffective. However, at our target BER of $10^{-5}$, the Soft-SIC scheme performs by about $3 \mathrm{~dB}$ better than the SUD-ID scheme.

Next, we consider the BER versus the number of users performance of the schemes under consideration in Fig. 3, where we assume $M=32 L=7$ and $E_{b} / N_{0}=15 d B$. We observe in Fig. 3 that for a high number of users, the performance of our proposed Soft-SIC scheme is poorer than that of the SUD-ID scheme. However, in the practical range of BERs, the Soft-SIC outperforms the ID scheme. The reason is that the Soft-SIC becomes overwhelmed, when the number of users is high. Thus, at high values of $N_{U}$, the best values of $\epsilon$ are those which ensure that all users' symbols are decoded without subjecting them to the reliability test discussed in Section III. By contrast, the iterative gain recorded in the context of the SUD-ID is achieved for all values of $N_{U}$ considered. However, it can be seen from Fig. 3 that at

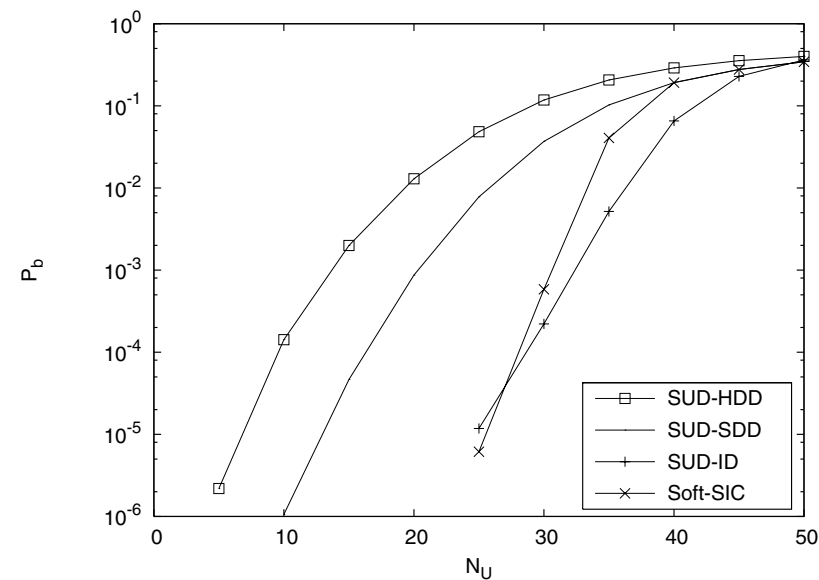

Fig. 4. BER versus number of users performance for SIC and ID schemes employed in soft decoded FFH-MFSK SLC receiver communicating over a Rayleigh fading channel, assuming $L=7, M=64$ and $E_{b} / N_{0}=15 \mathrm{~dB}$.

high $N_{U}$ values, the BER of all schemes considered becomes excessive to be of any practical use. By contrast, at the BER of $10^{-5}$, the Soft-SIC scheme is capable of supporting $50 \%$ more users than the SUD-ID scheme and nearly twice as many users as that supported by the SUD-SDD scheme.

In Fig. 4 we depict the BER versus the number of users performance of the schemes under consideration for higher order of modulation, i.e. for $M=64$. All other system parameters remain the same as in Fig. 3, except that the interleaver's block length is $60 \times 40$ bits. We observe in Fig. 4 that the SUD-ID scheme outperforms the Soft-SIC scheme for a wide range of $N_{U}$ values, although at BER of $10^{-5}$, the two schemes support a similar number of users. Comparison of this result to those shown in Fig. 3 demonstrates that the SUD-ID scheme exhibits more substantial performance benefits in the context of increased modulation orders. This observation is in harmony with the results of [13], where it has been shown that the iterative gain attained by the ID scheme increases as $M$ increases. By contrast, the performance of the SoftSIC scheme is mainly dependent upon its ability to cancel the interference and is hence not affected as substantially by the increased value of $M$, as that of the SUD-ID scheme.

The results of Fig. 5 characterize the effects of interleaver length (IL) on the BER performance of the schemes under consideration. All other parameters remain the same as in Fig. 4. The results of Fig. 5 indicate that the performance of the SUDID scheme is more adversely affected by a reduced IL than that of the Soft-SIC scheme. This is understandable, because the iterative gain of an ID scheme is strongly dependent upon the interleaver's size. Thus, as seen in Fig. 5, when $\mathrm{IL}=(10$ $\times 60$ ) is employed, the performance of the SUD-ID scheme degrades dramatically, while that of the Soft-SIC scheme is not much poorer compared to that at $\mathrm{IL}=(120 \times 200)$. This result indicates another advantage of our proposed scheme. More specifically, since having a high IL implies a longer delay, our Soft-SIC scheme is more suitable for applications requiring low delays.

Finally, in Fig. 6, we demonstrate the performance gain attained by the schemes under consideration, for various number of iterations. All other system parameters are the 


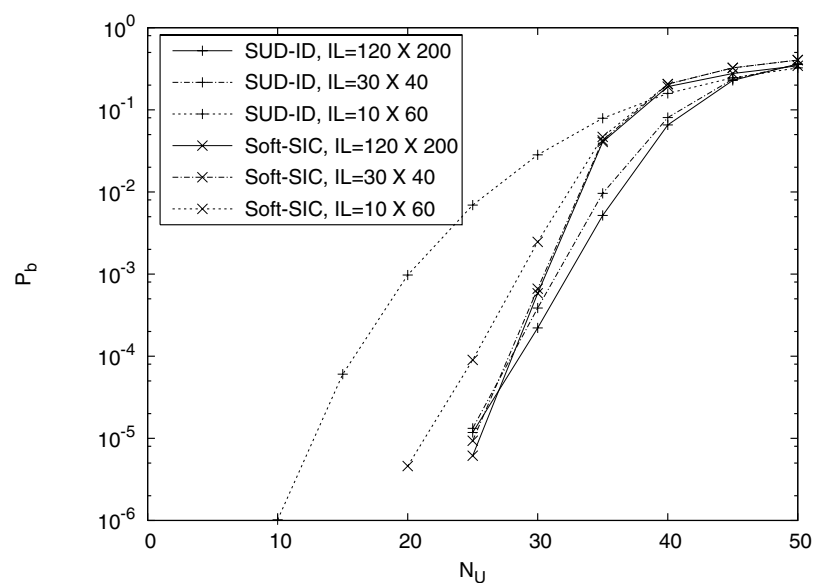

Fig. 5. BER versus number of users performance for SIC and ID schemes employed in soft decoded FFH-MFSK SLC receiver communicating over a Rayleigh fading channel for various values of interleaver sizes, assuming $L=$ $7, M=64$ and $E_{b} / N_{0}=15 \mathrm{~dB}$.

same as in Fig. 3. We observe that for the SUD-ID scheme, there is very little improvement after the second iteration, while our proposed Soft-SIC scheme yields noticeable BER improvements even during the fourth iteration. However, for low values of $N_{U}$, using an increased number of iterations does not yield further BER improvement.

\section{CONCLUSION}

We have investigated ID between a FFH-MFSK demodulator invoking SLC as well as a SISO binary convolutional decoding and derived the soft information to be passed from the demodulator to the channel decoder, when operating in Rayleigh fading MA channels. We also proposed a novel MUD scheme that invokes SIC in conjunction with SISO decoding. Our results shown in Figs. 2 to 6 demonstrate that the SDD using the derived soft information yields significant performance improvement over HDD, when subjected to MA interference. Furthermore, we have shown that the proposed Soft-SIC scheme is capable of substantially enhancing the achievable performance of the FFH-MFSK receiver. Our SoftSIC scheme outperforms the conventional SUD-ID scheme at a similar number of iterations, especially for $M \leq 32$, while in conjunction with $M>32$, the SUD-ID schemes benefits from a more substantial iterative gain. Moreover, the performance of the proposed Soft-SIC scheme is not significantly degraded, when a low IL is employed, rendering it superior to the SUD-ID scheme for low delay applications. The proposed Soft-SIC scheme also yields a higher iterative gain, as the number of iterations is increased, although the iterative gain saturates for both schemes after a certain number of iterations, when the number of users is low. Our future work will investigate the Soft-SIC scheme using a Recursive Systematic Convolutional (RSC) code and EXtrinsic Information Transfer (EXIT) charts. Although we investigated our Soft-SIC scheme using a binary convolutional coding scheme, the algorithm may also be employed in conjunction with other soft decoding schemes, such as non-binary LDPC codes, Reed Solomon (RS) codes or turbo codes. A specific drawback of the proposed scheme is that its achievable performance is dependent upon

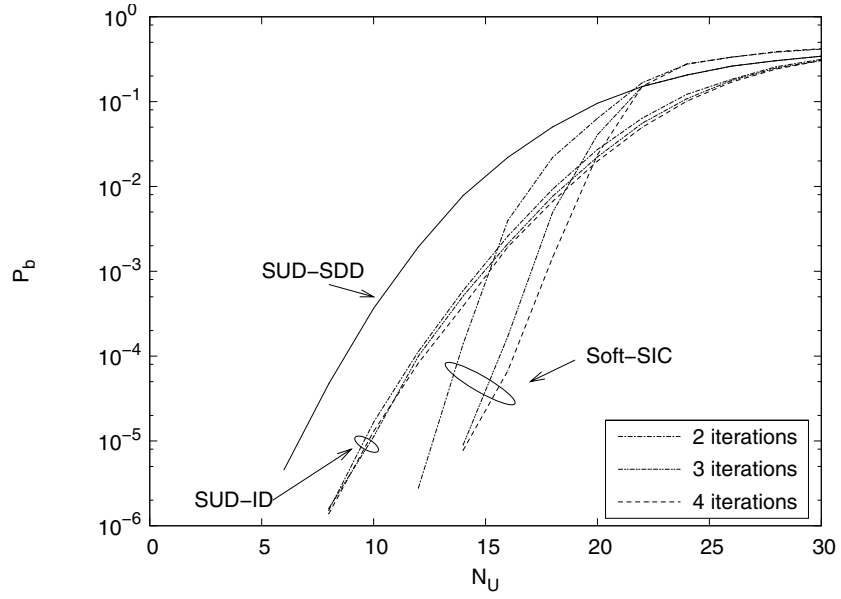

Fig. 6. BER versus number of users performance for SIC and ID schemes employed in soft decoded FFH-MFSK SLC receiver communicating over a Rayleigh fading channel for various number of iterations, assuming $L=7$, $M=32$ and $E_{b} / N_{0}=15 \mathrm{~dB}$.

the choice of the reliability test thresholds. In conclusion, for a wide range of parameter values of practical interest, our SoftSIC scheme proposed in Section III constitutes an attractive design option.

\section{REFERENCES}

[1] O. C. Yue, "Maximum likelihood combining for noncoherent and differentially coherent frequency hopping multiple access systems," IEEE Transactions on Information Theory, vol. IT-28, pp. 631-639, July 1982.

[2] U. C. Fiebig, "Iterative interference cancellation for FFH/MFSK MA systems," in IEEE Proceedings of International Conference on Cоттиnications, pp. 90-95, December 1996.

[3] J. G. Proakis, Digital communications. Singapore: Mcgraw-Hill, 2001.

[4] L. Hanzo, L.-L. Yang, E.-L. Kuan, and K. Yen, Single- and Multi-Carrier DS-CDMA: Multi-User Detection, Space-Time Spreading, Synchronisation, Networking and Standards. New York: John Wiley and sons, England, 2003.

[5] J. Hagenauer, "Forward error correcting for CDMA systems," in IEEE 4th International Symposium on Spread Spectrum Techniques and Applications Proceedings, 1996, vol. 2, pp. 566 - 569, September 2001.

[6] H. V. Poor, "Iterative multiuser detection," IEEE Signal Processing Magazine, vol. 21, pp. 81 - 88, January 2004.

[7] Y. T. Su and R. C. Chang, "Performance of fast FH/MFSK Signals in jammed binary channels," IEEE Transactions on Communications, vol. 42, pp. 2414 - 2422, July 1994.

[8] B. M. Peric, M. R. Souryal, E. Larsson, and B. R. Vojcic, "Soft Decision Metrics for Turbo-coded FH M-FSK Ad Hoc Packet Radio Networks," in IEEE 61st Vehicular Technology Conference, Spring 2005, vol. 2, pp. 724 - 727, May-June 2005.

[9] N. Sharma and E. Geraniotis, "Soft multiuser demodulation and iterative decoding for FH/SSMA with a block turbo code," IEEE Transactions on Communications, vol. 51, pp. 1561 - 1570, September 2003.

[10] U. C. Fiebig, "Soft-decision and erasure decoding in fast frequencyhopping systems with convolutional, turbo, and Reed-Solomon codes," IEEE Transactions on Communications, vol. 47, pp. 1646 - 1654, November 1999.

[11] D. Park and B. G. Lee, "Iterative decoding in convolutionally and turbo coded MFSK/FH-SSMA systems," in IEEE International Conference on Communications, ICC 2001, vol. 9, pp. 2784 - 2788, June 2001.

[12] P. C. P. Liang and W. E. Stark, "Algorithm for joint decoding of turbo codes and M-ary orthogonal modulation," in IEEE International Symposium on Information Theory, 2000, p. 191, June 2000.

[13] M. C. Valenti, E. Hueffmeier, B. Bogusch, and J. Fryer, "Towards the capacity of noncoherent orthogonal modulation: BICM-ID for turbo coded NFSK," in IEEE Conference on Military Communications, 2004. MILCOM 2004, vol. 3, pp. 1549 - 1555, October-November 2004.

[14] L. Hanzo, T. H. Liew, and B. L. Yeap, Turbo Coding, Turbo Equalisation and Space-Time Coding for Transmission over Fading Channels. England: John Wiley and sons, England, 2002. 